\title{
PRODUÇÃO DE LIPASE BRUTA PELO FUNGO Rhizopus sp E AVALIAÇÃO DAS PROPRIEDADES CATALÍTICAS EM REAÇÕES DE INTERESTERIFICAÇÃ̃.
}

\author{
P. SPERANZA ${ }^{1}$, T. S. N. GOMES ${ }^{2}$, G. A. MACEDO ${ }^{1}$ \\ ${ }^{1}$ Universidade Estadual de Campinas, Faculdade de Engenharia Alimentos \\ ${ }^{2}$ Universidade Estadual de Campinas, Faculdade de Engenharia Química \\ E-mail para contato: tauangomes@yahoo.com.br
}

\begin{abstract}
RESUMO - O fungo Rhizopus sp foi repicado para a produção de lipase por fermentação em estado sólido. Na fermentação utilizou-se farelo de trigo como substrato. Após 72 horas, o fermentado foi semi purificado utilizando-se sulfato de amônio, concentrado por diálise e liofilizado. A atividade lipolítica deste extrato liofilizado foi de $8,0 \mathrm{U} / \mathrm{g}$. O extrato liofilizado foi utilizado como catalizador para a interesterificação enzimática entre o óleo de buriti e a gordura de murumuru e o óleo de patauá e a estearina de palma. A enzima foi capaz de catalisar a reação, causando uma redistribuição dos ácidos graxos no triacilglicerol.
\end{abstract}

\section{INTRODUÇÃO}

Lipase é um grupo de enzimas cuja função é catalisar a hidrólise de triacilgliceróis, produzindo ácidos graxos livres, diacilgliceróis, monoacilgliceróis e gliceróis. Sendo essas reações reversíveis na presença da enzima, a indústria a utiliza para biotransformações como interesterificações e transesterificações, aplicando na produção de detergentes, aromas com bases lipídicas e transformações gerais em óleos. O potencial das enzimas se deve ao fato de poderem ser produzidas em larga escada por fermentação microbiana, facilitando a utilização pela indústria (Macedo, 1995).

Interesterificação é a redistribuição dos ácidos graxos no triacilglicerol, modificando então suas propriedades físico-químicas (Kaki et. al, 2014). A interesterificação pode ser feita quimicamente ou enzimaticamente. A química troca as posições dos ácidos graxos de modo randômico, já a enzimática pode redistribuir randomicamente ou de modo específico, dependendo da regioseletividade. No entanto, a interesterificação enzimática requer condições de reações mais suaves que a química para que ocorram menos reações indesejadas, diminuir o pós-tratamento e, consequentemente, prevenir a perda de óleo (Costales-Rodríguez et. al, 2008).

A utilização de lipases é preferível à interesterificação química, pois estas possuem estruturas estereoespaciais específicas e os produtos finais podem ser melhores controlados. A interesterificação geralmente utiliza-se de dois tipos de triacilgliceróis, um óleo, em estado líquido, e gordura, em estado sólido ou semissólido (Kaki et. al, 2014). 
Esta técnica é ótima para aumentar o valor comercial dos óleos e nisso os lipídeos amazônicos de destacam. Existem óleos pouco explorados e seus estudos possibilitam uma maior gama de variedade de produtos e lipídeos com novas características (Cunha et. al, 2014).

\section{OBJETIVO}

Objetivou-se o aprendizado das técnicas de repicagem do fungo Rhizopus sp, a fermentação em estado sólido, a semi-purificação do extrato bruto fermentado, a liofilização e a avaliação da atividade lipolítica do extrato bruto. Além disso, avaliou-se a atividade catalítica desta lipase bruta em reações de interesterificação de óleos provenientes da Amazônia.

\section{MATERIAL E MÉTODOS}

\subsection{Repicagem do Fungo Rhizopus sp}

Pesou-se em balança semi-analítica PDA (Potato Dextrose Agar) em proporção 3,9 g/100 mL de água destilada. Aqueceu-se até começo de ebulição e adquirir transparência, depois transferiu-se cerca de $10 \mathrm{~mL}$ da solução para tubos de ensaio. Então, fechou-se os tubos de ensaio com tampão e envolve-os com papel kraft, levando-os para autoclavagem a $121^{\circ} \mathrm{C}$ por 15 minutos.

Após retirado da autoclave, deixou-se os tubos inclinados até endurecimento do meio. De modo a manter a assepsia, pegou-se a alça de platina já flambada e fria e raspou-se os esporos dos tubos de ensaio já com crescimento e os passou para os tubos autoclavados. Então, colocou-se em estufa a $30^{\circ} \mathrm{C}$ por 72 horas.

\subsection{Produção de lipase por Rhizopus sp}

3.2.1. Preparo do meio: Preparou-se uma mistura de farelo de trigo e água destilada na proporção 60:40 em peso e em erlenmeyers de $500 \mathrm{~mL}$ pesou-se $20 \mathrm{~g}$ da mistura. Então levou-se para autoclave a $121^{\circ} \mathrm{C}$ por 15 minutos.

3.2.2. Inoculação no meio: Adicionou-se $5 \mathrm{~mL}$ de água destilada estéril nos tubos com cultura microbiana de Rhizopus sp. e raspou-se com alça de platina fazendo os esporos se misturarem na água. Então, retirou-se $1 \mathrm{~mL}$ da suspenção de esporos e inoculou-se nos erlenmeyers com meio de trigo e água destilada. Colocou-se os erlenmeyers em estufa a $30^{\circ} \mathrm{C}$ por 120 horas.

3.2.3. Extração da enzima: Colocou-se $100 \mathrm{~mL}$ de água destilada com uma proveta nos erlenmeyers e homogeneizou-se com bastão de silicone deixando em repouso por 1 hora com ocasional agitação. Levou-se os erlenmeyers de $500 \mathrm{~mL}$ com funil e gaze para a sala de inoculação e colocou-se máscara, luvas e óculos de proteção para fazer as etapas. 
Despejou-se no funil com gaze o conteúdo dos erlenmeyers pressionando com as mãos, de modo a obter o maior filtrado possível. Esterilizou-se em autoclave os resíduos e os erlenmeyers sujos para descarte e lavagem de vidrarias. Colocou-se o líquido filtrado em proveta para medir o volume e adicionou-se sulfato de amônio em proporção de $560 \mathrm{~g} / \mathrm{L}$ enquanto toda a solução ficou em banho de gelo e com misturador de hélice ligado a baixa velocidade. Esta etapa exige-se muito cuidado, pois não pode formar espuma, então colocouse o sal devagar e mexeu-se com um bastão de vidro para tirar grumos e grânulos.

Colocou-se papel alumínio no recipiente e guardo-o em geladeira até o dia seguinte. Colocou-se 2 galões de água destilada na geladeira para o dia seguinte. Pesou-se nos recipientes da centrífuga fazendo os pares de modo equilibrado. Ligou-se a centrífuga a 10000 rpm a $4^{\circ} \mathrm{C}$ por 15 minutos.

Desprezou-se o sobrenadante e recolheu-se o precipitado com espátula e pouca água destilada. Pegou-se o saco plástico de celulose para dialise, molhou-se em água para facilitar abri-lo e molhou-se 2 barbantes cumpridos. Fechou-se a parte de baixo do saco para diálise com um barbante a aproximadamente 3 dedos da base, dando vários nós fortes. Lavou-se o interior com água destilada.

Colocou-se o precipitado dentro do saco de diálise com a ajuda de um funil e fechou-se com o outro barbante do mesmo modo, deixando espaço de ar dentro do saco. Colocou-se a água destilada gelada em um balde (deixada na geladeira) e mergulhou-se no balde, de modo a deixar o saco inteiramente dentro da água. Deixou-se em dialise, colocando em geladeira por 48 horas, trocando a água destilada de 2 a 3 vezes por dia (observando a mudança de coloração da água).

3.2.4. Liofilização: Após a dialise, colocou-se o conteúdo do saco de diálise em placas plásticas, com furos na tampa e deixou-se em congelador por pelo menos 24 horas. Ligou-se o liofilizador na tomada, fechou-se a válvula lateral e ligou-se o botão "frio". Deixou-se atingir a temperatura de $-42^{\circ} \mathrm{C}$ e colocou-se as placas nas bandejas rapidamente, sem deixar descongelar. Ligou-se o vácuo segurando a tampa até ouvir o barulho diminuir. Depois de duas horas abriu-se a válvula do motor e passando novamente duas horas, fechou-se a mesma válvula. Deixou-se liofilizando por 48 horas e por fim guardou-se a enzima já liofilizada em potes plásticos no congelador.

\subsection{Atividade enzimática da lipase (atividade lipolítica)}

Fez-se uma emulsão de óleo de oliva extra virgem e goma arábica 7\% (25:75 v/v) utilizando turrax por 5 minutos. Então montou-se um sistema em erlenmeyers de $125 \mathrm{~mL}$ de 5 $\mathrm{mL}$ de emulsão, $2 \mathrm{~mL}$ de tampão fosfato $\mathrm{pH} 7,00,1 \mathrm{M}$ e $1 \mathrm{~mL}$ de solução enzimática na concentração de $10 \mathrm{mg} / \mathrm{mL}$ de enzima.

Incubou-se os erlenmeyers em banho-maria a $37^{\circ} \mathrm{C}$ por 30 minutos com agitação de $130 \mathrm{rpm}$. Então, paralisou-se a reação adicionando $15 \mathrm{~mL}$ de solução acetona:etanol (1:1 v/v). Adicionou-se três gotas de fenolftaleína e titulou-se com hidróxido de sódio $(\mathrm{NaOH}) 0,05 \mathrm{M}$ que reagiram com os ácidos graxos liberados e então com o indicador na primeira gota de excesso, deixando a solução levemente rosa. 


\subsection{Interesterificação enzimática}

Para os testes de interesterificação utilizou-se óleo de buriti, óleo de patauá e gordura de murumuru comprados em mercado local na cidade de Belém (Pará, Brasil). Também utilizou-se estearina de palma cedido pela Agropalma (Pará, Brasil). A lipase de Rhizopus sp. foi produzida no laboratório pelo método citado nos itens anteriores e utilizou-se lipase comercial imobilizada (Lipozyme TL-IM) suprido por Novozymes.

Para os testes utilizou-se dois tipos de misturas, óleo de buriti com gordura de murumuru e óleo de patauá com estearina de palma, ambos na proporção $70: 30(\mathrm{~m} / \mathrm{m})$. As reações foram feitas com três tipos de sistemas enzimático: lipase comercial (TL), lipase de Rhizopus sp. e uma mistura igual das duas enzimas. Para a mistura do substrato utilizou-se 10 g e a concentração das enzimas variou de 2,5 a $10 \%(\mathrm{~m} / \mathrm{m})$. Todos os testes foram feitos em banho-maria sob vácuo a $150 \mathrm{rpm}$ e por 24 horas, variando somente a temperatura, $40^{\circ} \mathrm{C}$ para a mistura óleo de buriti com gordura de murumuru e $50^{\circ} \mathrm{C}$ para a mistura de óleo de patauá com estearina de palma.

Após a reação os produtos foram imediatamente filtrados usando uma membrana de $0,45 \mu \mathrm{m}$ e então congelados com nitrogênio. Depois eliminou-se os ácidos graxos livres e os acilgliceróis colocando cuidadosamente álcool aquecido a $50^{\circ} \mathrm{C}$ na parte superior dos eppenrdofs e os deixando de molho a $50^{\circ} \mathrm{C}$ por 30 minutos, fazendo com que os compostos indesejados migrassem para o etanol e então retirando o etanol junto com estes compostos. Repetiu-se o procedimento 3 vezes. Os eppendorfs foram embalados com papel alumínio e colocou-se nitrogênio antes de congela-los novamente.

\subsection{Análise de triacilglicerol}

A composição de ácidos graxos foi usada para prever os grupos de triacilgliceróis na mistura não interesterificada com o software PrÓleos, o qual usa algoritmo matemático que descreve a distribuição dos ácidos graxos nas moléculas de triacilglicerol (Antoniosi Filho et al., 1995). A composição do triacilglicerol presente nos lipídeos interesterificado foi analisada de acordo com 1,3-randomizado, 2-teóricamente randômico (distribuição não randômica), e 1,2,3-teóricamente randômico (distribuição randômica), baseado na análise de distribuição regioespecífica (D'Agostini and Gioielli, 2002; Guedes et al., 2014).

\section{RESULTADOS E DISCUSSÃO}

\subsection{Atividade Lipolítica}

A atividade lipolítica da enzima produzida por fermentação em estado sólido sólida foi de 8,0 U/g. Comparando-se o resultado obtido da lipase comercial Lipozyme TL-IM (Novozyme) e a lipase comercial lipozyme RM-IM (Novozyme), purificadas e imobilizadas (Figura 1) (Anschau et.al, 2009) é possível verificar que a atividade da enzima Rhizopus sp. foi menor que a da comercial TL-IM, porém maior que a comercial RM-IM.

A enzima de Rhizopus sp. produzida não foi totalmente purificada nem imobilizada como as comerciais, portanto sua atividade lipolítica poderia ser maior se passasse por estes 
processos. Esta enzima mostrou-se promissora para aplicações, como por exemplo, na interesterificação enzimática de óleos.

Figura 1 - Atividade enzimática da enzima produzida por Rhizopus sp e comerciais da literatura.

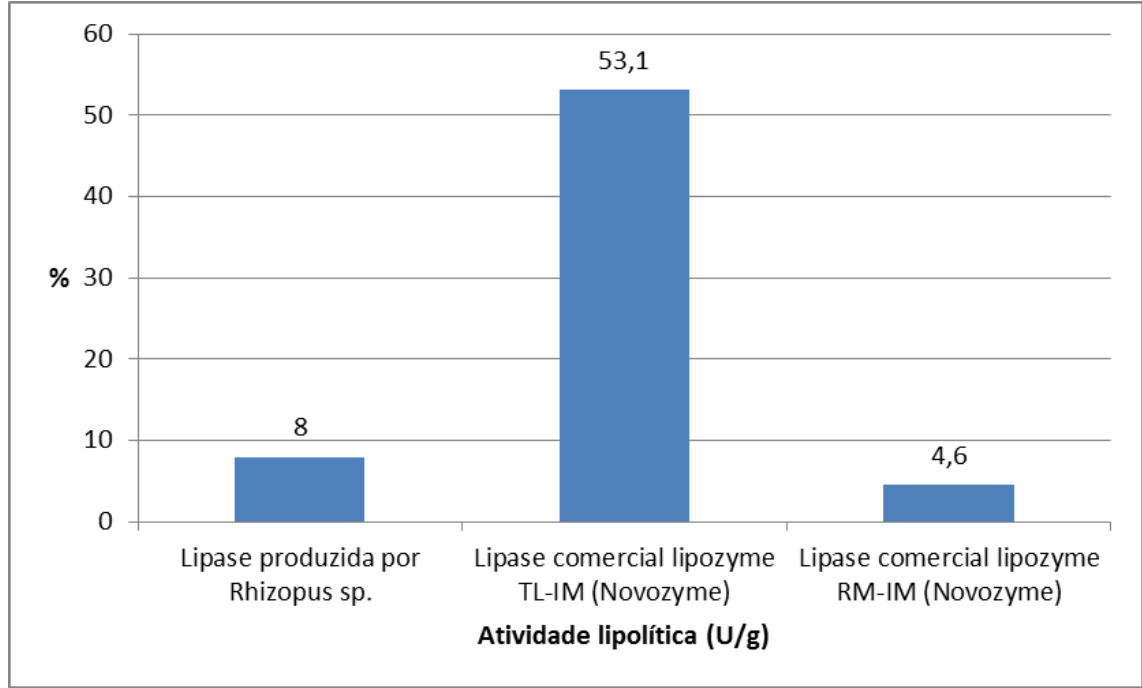

\subsection{Interesterificação}

Pode-se reparar que os óleos (buriti e óleo de palma) tem mais ácidos graxos insaturados e as gorduras (murumuru e estearina de palma) tem uma maior porcentagem de ácidos graxos saturados (Tabela 1). As misturas dos lipídeos contêm uma porcentagem parecidas dos quatro tipos de triacilgliceróis. Após a interesterificação a porcentagem foi alterada, indicando que a enzima atuou nos ácidos graxos e constituiu mais triacilgliceróis mono e di-insaturados.

Tabela 1 - Porcentagem de ácidos graxos saturados (S) e insaturados (I) para cada lipídeo

\begin{tabular}{lcccc|cccc}
\hline TAG & $\begin{array}{c}\text { Buriti } \\
(\%)\end{array}$ & $\begin{array}{c}\text { Murumuru } \\
(\%)\end{array}$ & $\begin{array}{c}\text { Mistura não } \\
\text { interesterificada } \\
(\%)\end{array}$ & $\begin{array}{c}\text { Óleo } \\
\text { interesterificado } \\
(\%)\end{array}$ & $\begin{array}{c}\text { Óleo de } \\
\text { Patauá } \\
(\%)\end{array}$ & $\begin{array}{c}\text { Estearina } \\
\text { de Palma } \\
(\%)\end{array}$ & $\begin{array}{c}\text { Mistura não } \\
\text { interesterificada } \\
(\%)\end{array}$ & $\begin{array}{c}\text { Óleo } \\
\text { interesterificado } \\
(\%)\end{array}$ \\
\hline SSS & 0.9 & 69.7 & 21.5 & 6.0 & 0.7 & 20.5 & 6.5 & 8.9 \\
S.I & 9.8 & 25.6 & 14.4 & 28.6 & 8.7 & 41.7 & 18.6 & 33.1 \\
SI 2 & 39.3 & 4.3 & 28.8 & 43.7 & 37.2 & 29.0 & 34.8 & 41.1 \\
III & 50.0 & 0.5 & 35.3 & 21.7 & 53.2 & 8.5 & 39.9 & 16.9 \\
\hline
\end{tabular}

A análise de triacilglicerol indicou que, após a interesterificação, os grupos de triacilgliceróis foram modificados, o que remete o efeito da enzima na modificação de ácidos graxos do glicerol. 


\section{Conclusão}

O fungo de Rhizopus sp foi capaz de produzir lipase quando fermentado em um meio semi-sólido. O extrato bruto liofilizado obtido, mostrou-se eficiente como catalisador para a reação de interesterificação de óleos da Amazônia, sendo capaz de redistribuir os ácidos graxos no triacilglicerol. Esta enzima tem grande potencial de aplicação nestas reações.

\section{Referências Bibliográficas}

ANSCHAU, A.; ARAGÃO, V. C.; BURKET, C. A. V.; BURKET, J. F. M; KALIL, S. J.; PORCIUNCULA, B. D. A; THIESEN, C. Síntese enzimática de butirato e isoamila empregando lipases microbianas comerciais. Quim. Nova, v. 32, n. 9, 2268-2272, 2009.

ANTONIOSI, FILHO, N. R.; MENDES, O. L.; \& LANÇAS, F. M. (1995). Computer prediction of triacylglycerol composition of vegetable oils by HRGC. Chromatographia, 40, 557-562.

COSTALES-RODRÍGUEZ, R.; GIBON, V.; GREYT, W.; VERHÉ, R. Chemical and Enzymatic Interesterification of a Blend of Palm Stearin: Soybean Oil for Low transMargarine Formulation. J Am Oil Chem Soc, 2009.

CUNHA, R. L.; MACEDO, G. A.; MACEDO, J. A.; RIBEIRO, A. P. B.; SPERANZA, $P$. Influence of emulsion droplet size on antimicrobial activity of interesterified Amazonian oils. LWT - Food Science and Technology, 2014.

D'AGOSTINI, D.; GIOIELLI, L. A. Sterospecific distribution of structured lipids obtained from palm oil, palm kernel oil and medium chain triacylglycerols. Brazilian Journal of Pharmaceutical Sciences, 38, 345-354, 2002.

GUEDES, A. M. M.; MING, C. C.; RIBEIRO, A. P. B.; SILVA, R. C.; GIOIELLI, L. A.; GONÇALVES, L. A. G. Physicochemical Properties of Interesterifield blends of Fully Hydrogenated Crambe abyssinica Oil and Soybean Oil. Journal of the American Oil Chesmists' Society, 91, 111-123, 2014.

KAKI, S. S.; NEEHARIKA, T. S. V. R.; PRASAD, R. B. N.; RAGINI, Y.; RALLABANDI, R.; RANI, K. N. P. Lipase catalyzed interesterification of rice bran oil with hydrogenated cottonseed oil to produce trans free fat. Journal of Food Science and Technology, 2014.

MACEDO, G. A. Produção, purificação e caracterização bioquímica e aplicações de lipase de Geotrichum sp. Tese (mestrado em ciência dos alimentos), Faculdade de Engenharia de Alimentos (FEA), Universidade Estadual de Campinas (UNICAMP), 1995. 